\title{
AN EVALUATION OF THE SINGLE ORIENTATION METHOD FOR TEXTURE DETERMINATION IN MATERIALS OF MODERATE TEXTURE STRENGTH
}

\author{
STUART I. WRIGHT and BRENT L. ADAMS \\ Department of Mechanical Engineering, Yale University, New Haven, \\ CT 06520 USA
}

(Received 15 January 1989)

\begin{abstract}
New microscopy technology has made it feasible to consider using single orientation measurements to obtain Orientation Distribution Functions (ODF). Single orientations are preferred over pole figure data in ODF calculations because the odd terms of the series expansion are not directly accessible when pole figures are used. However, the number of single orientation measurements required to calculate a statistically reliable ODF has generally been considered too large to practically obtain. This study found that the location of the major features of the ODF in rolled aluminum sheet were identifiable after only 100 measurements. However, the heights of the peaks and the secondary features required many more measurements to stabilize. Unless the orientation measurements can be automated or orientation correlation data is needed the pole figure method remains a more efficient means of obtaining the data necessary to measure texture.
\end{abstract}

KEY WORDS Single orientation method, ODF analysis, scanning electron microscopy.

\section{INTRODUCTION}

The experimental determination of the Orientation Distribution Function (ODF) is ideally obtained by taking a material apart grain by grain and measuring the orientation and volume of each grain. This experiment is practically impossible and, therefore, more experimentally feasible experiments have been devised to facilitate the determination of the ODF. The most common of these is the pole figure technique. However, with new computer aided microscopy it has become practical to consider measuring single orientations in a sample using the analysis of electron backscattering patterns. The purpose of this study was to investigate the possibility of using single orientations to calculate statistically accurate ODFs and to compare this method with the traditional pole figure method.

ODFs calculated using classical pole figure techniques are incomplete due to the absence of odd terms in the series expansion. Approximation methods have been used to resolve the ambiguity of the odd coefficients in ODFs calculated using pole figures. Gaussian fitting (Lücke, 1981) has been used to approximate coefficients but requires additional assumptions about the form of the complete ODF. Bunge has proposed a technique for calculating the odd coefficients by enforcing posivity in the pole figures and the ODF (Dahms and Bunge, 1988). This method requires no additional assumptions about the form of the ODF and has, therefore, been used in this study. 
The single orientation method gives immediate access to the odd and even terms of the ODF without any corrective measures. However, collecting statistically reliable data sets may be prohibitively time consuming. The strength of the texture also determines the number of orientations required to calculate a statistically reliable ODF; the more random the texture the more measurements required.

This study examines some of the issues surrounding the use of the single orientation method. One thousand single orientation measurements were taken on a piece of rolled aluminum which exhibited a moderate-strength texture. ODFs were calculated following the formalism of Bunge (1982) using 100, 200, $300, \ldots$ and 1000 measurements. A set of ODFs were calculated using both the odd and even terms of the series expansion and a second set of ODFs were calculated omitting the odd coefficients to simulate a pole figure experiment. Positivity was enforced in the second set of ODFs to give approximations of the complete ODFs. An ODF was also constructed using $\mathrm{X}$-ray pole figures. Comparisons were made to examine the accuracy of ODFs constructed using single orientation data.

\section{THEORETICAL DEVELOPMENT}

The well known expansion of the ODF into a series of generalized spherical harmonics (Bunge, 1965) is given by:

$$
f(g)=\sum_{l=0}^{\infty} \sum_{\mu=0}^{M(l)} \sum_{v=0}^{N(l)} C_{l}^{\mu v} \dot{T}_{l}^{\mu v}(g)
$$

The $C$ coefficients can be obtained by either measuring single orientations or by using X-ray diffraction data in the form of pole figures.

\section{Single Orientation Method}

The coefficients can be calculated using single orientation measurements (Bunge, 1965) by the formula

$$
C_{l}^{\mu \nu}=(2 l+1)(1 / N) \sum_{i=0}^{N} \dot{T}_{l}^{* \mu \nu}\left(g_{i}\right)
$$

This is essentially equivalent to situating a Dirac delta function at the orientation $g_{i}$ in Euler space for each of the $N$ measurements. This method generates a negative region in the ODF surrounding the orientation $g_{i}$. Using Gaussian functions instead of Dirac functions to represent the measurements results in a more correct ODF. The Gaussian formulation (Wagner et al., 1981) is given by

$$
C_{l}^{\mu \nu}=(1 / N) \sum_{i=0}^{N} K \dot{T}_{l}^{* \mu \nu}\left(g_{i}\right)
$$

where

$$
K=\frac{\exp \left(-l^{2} \Phi_{0}^{2} / 4\right)-\exp \left(-(l+1)^{2} \Phi_{0}^{2} / 4\right)}{1-\exp \left(-\Phi_{0}^{2} / 4\right)}
$$


where $\Phi_{0}$ is the 1/e-width of the Gaussian peaks located at each $g_{i}$. If Euler space is divided up into "spheres" of radius $\Phi_{0}$ then the volume, $v$, of these spheres is given by

$$
v=\int_{0}^{\Phi_{0}} \sin ^{2}(\Phi / 2) 8 \pi d \Phi=4 \pi\left(\Phi_{0}-\sin \Phi_{0}\right)
$$

In order to have $p$ points in each "sphere", $\Phi_{0}$ must be calculated using the equality

$$
(N / p) v=V_{0}
$$

where $N$ is the number of individual crystal measurements and $V_{0}$ is the volume of the unit cell. For cubic symmetry this expression can be rewritten as

$$
(N / p) 4 \pi\left(\Phi_{0}-\sin \Phi_{0}\right)=(1 / 24) 8 \pi^{2} .
$$

If $\Phi_{0}$ is not too large then sin $\Phi_{0}$ can be replaced by $\Phi_{0}-\Phi_{0} / 3$ ! and Eq. (7) can be solved for $\Phi_{0}$.

$$
\Phi_{0} \approx(\pi p / 2 N)^{1 / 3} .
$$

For a random texture there must be at least one point in each sphere and $p$ must, therefore, be at least 1 . For stronger textures the points will no longer be distributed equally throughout Euler space. For intermediate textures, which is the case in the sample being examined, $p \approx 0.5$.

\section{Pole Figure Method}

The $C$ coefficients are obtained by the solution of the following equation when pole figures are being used (Bunge, 1965):

$$
P_{\mathbf{h}_{i}}(\mathbf{y})=\sum_{l=0}^{\infty} \sum_{\mu=0}^{M(l)} \sum_{v=0}^{N(l)} C_{l}^{\mu v} k_{l}^{* \mu}\left(\mathbf{h}_{i}\right) \dot{k}_{l}^{v}(\mathbf{y})
$$

where the $\dot{k}_{l}^{* \mu}\left(\mathbf{h}_{i}\right)$ and $\dot{k}_{l}^{\nu}(\mathbf{y})$ are symmetric spherical surface harmonics and the asterisk denotes the complex conjugate. $P_{\mathbf{h}_{i}}(\mathbf{y})$ is the pole figure, the $\mathbf{h}_{i}$ denoting the Miller indices, $(h k l)$, of the $i$ th pole figure and $\mathbf{y}$ representing the direction of the pole in the sample reference system in spherical polar coordinates. Bragg's law for diffracted waves requires that the normal of the scattering plane be fixed in space but not direction (sign) (Matthies, 1979). This means that all diffraction experiments are centrosymmetric and can, therefore, not discriminate between the $+h$ and $-h$ crystal directions (Bunge, 1982). The virtual pole figure, $\tilde{P}_{\mathbf{h}_{i}}(\mathbf{y})$ is then the superposition of the pole figures of the equivalent directions $-h$ and $+h$ and can be expressed as

$$
\tilde{P}_{\mathrm{h}}(\mathbf{y})=1 / 2\left[P_{+\mathrm{h}}(\mathbf{y})+P_{-\mathrm{h}}(\mathbf{y})\right] .
$$

Equation (9) can be written as

$$
\tilde{P}_{\mathbf{h}_{i}}(\mathbf{y})=\sum_{l=0}^{\infty} \sum_{\mu=0}^{M(l)} \sum_{v=0}^{N(l)} \tilde{C}_{l}^{\mu v} \dot{k}_{l}^{* \mu}\left(\mathbf{h}_{i}\right) \dot{k}_{l}^{v}(\mathbf{y})
$$


where the coefficients, $\tilde{C}_{l}^{\mu \nu}$, define the virtual texture and take on the form

$$
\tilde{C}_{l}^{\mu v}=C_{l}^{\mu v} 1 / 2\left[1+(-1)^{l}\right]= \begin{cases}C_{l}^{\mu v} & \text { for } l \text { even } \\ 0 & \text { for } l \text { odd }\end{cases}
$$

This means that the odd part of the ODF cannot be obtained from pole figure measurement.

Using Gaussian spread functions to approximate ODFs, Lücke et al. (1976), observed that false maxima appeared in ODFs reproduced from pole figures. This was thought to be due to truncation error in the series expansion, however, Matthies (1979) has shown that these so-called "ghost peaks" are due to the absence of odd terms in the series expansion.

Methods other than the harmonic have been proposed for determining ODFs, including the Vector (Ruer, 1976; Vadon, 1981) and WIMV (Matthies and Vinel 1982; Williams 1968a, b; Imhof, 1982) methods; however, the harmonic method remains the most popular method for ODF calculation in higher symmetry materials. Methods have, therefore, been proposed to resolve the ambiguity of the odd coefficients. The most notable are, the application of Gauss-type models (Lücke et al., 1981); enforcing non-negativity using a quadratic form (Van Houtte, 1983) and the zero-range method coupled with the positivity method (Dahms and Bunge, 1988). This study employs a positivity method adapted from Dahms and Bunge outlined in the following paragraph.

The ODF is a probability density function and must, therefore, be positive over all orientations. Enforcing positivity in the pole figures makes the experimentally obtained even coefficients more reliable; whereas, enforcing positivity in the ODF provides a means for obtaining the odd coefficients. Since single orientation data was used to obtain the even coefficients in this study, it was not necessary to enforce positivity in the pole figures to get a more reliable set of even coefficients.

Since the symmetric generalized spherical harmonics form an orthonormal set of functions, the $C$ coefficients can be determined when an ODF is known per

$$
C_{l}^{\mu v}=(2 l+1) \oint f(g) \dot{\tilde{T}}_{l}^{* \mu v}(g) d g .
$$

Let the ODF be separated into two parts

$$
f(g)=\tilde{f}(g)+\tilde{f}(g)
$$

where $f(g)$ represents the true complete ODF, $\tilde{f}(g)$ represents the incomplete ODF calculated using even coefficients only and $\tilde{f}(g)$ represents the ODF calculated using only the odd coefficients. Let $\bar{f}(g)$, represent an ODF constructed by setting all positive regions of $\tilde{f}(g)$ to zero and then taking the absolute value of the remaining negative regions. In mathematical terms

$$
\bar{f}(g)=1 / 2(|\tilde{f}(g)|-\tilde{f}(g)) .
$$

$\tilde{f}(g)$ can now be used in Eq. (13) to calculate a set of odd coefficients which fit this function. These coefficients can be used along with the original coefficients to calculate a corrected ODF. This procedure can be repeated until the corrected ODF, $f^{(i)}(g)$, ( $i$ represents the $i$ th iteration), contains no more negative regions. One of the advantages of this technique is that the experimentally obtained even coefficients are retained. 


\section{PROCEDURE}

\section{Single Orientations}

Electron backscattering diffraction produces patterns which allow one to identify the rotation of a grain in which the electron beam is currently located with respect to some macroscopic coordinate axes. Dingley (1988) has designed a camera which transmits the image of the backscattered electrons on a phosphor screen placed in the specimen chamber to a computer. The computer has software residing on it for analyzing the patterns with help from the user to identify the (112) and (111) or (114) zone axes. The precision of this system is approximately $1.0^{\circ}$.

An approximately ten $\mathrm{cm}$ by five $\mathrm{cm}$ piece was shear cut from a sheet of rolled $1100(99 \% \mathrm{Al})$ aluminum obtained from ALCOA. This was then cut into a $2.5 \mathrm{~cm}$ by $2.5 \mathrm{~cm}$ piece using an abrasive saw to minimize deformation. No mechanical polishing was performed in order to avoid introducing any tilt on the surface of the sample. The specimen was then electro-polished and etched enabling the grain boundaries to be identified on the SEM which aided in the selection of a magnification that allowed approximately fifty grains to fill the screen of the SEM. This occurred at $1000 \mathrm{x}$ magnification. An array of fifty points was then systematically followed on the screen and an orientation measurement made at each point. Electron backscattering requires that the sample be tilted $70^{\circ}$. This means that the image of the sample on the microscope screen in scan mode is also tilted $70^{\circ}$. Since the measurement array is created from the screen, a square array would be projected on to the sample as a rectangular array. The measurement array was constructed to correct for the tilt in order to obtain a measurement array on the specimen with a one to one aspect ratio. The resulting aspect ratio was one to 1.16. After fifty measurements were completed the sample was translated and a new location was used to make another set of fifty measurements. One thousand measurements were made using this procedure.

Using this data ODFs were calculated using 100,200,300, . and 1000 measurements. Cubic sample symmetry was enforced and the ODFs were calculated to $l=22$. Let the ODF calculated using $N$ measurements be denoted by $f_{N}(g)$. Pole figure experiments were simulated by calculating ODFs as described above while excluding the odd coefficients. These ODFs were then corrected using the positivity technique described previously. Let these ODFs be represented by $f_{N}^{(i)}(g)$ where $(i)$ represents the number of correction iterations the incomplete ODF has undergone.

\section{Pole Figure Measurement}

Pole figures were measured on the aluminum sheet using X-ray diffraction and an ODF calculated from this data using Roe's (1965) formalism at the ALCOA research center. Using the $W$ coefficients this ODF was calculated in the identical subregion of Euler space as were the ODFs calculated from single orientation measurements. Cubic sample symmetry and orthorhombic processing symmetry were enforced and the ODF was expanded to $l=22$. Let this ODF be denoted by $f_{\text {polef. }}(g)$. 


\section{Mean Square Error Measurement}

A mean square error measurement was employed to describe the difference between two ODFs $f_{a}(g)$ and $f_{b}(g)$. This is given by the following equation:

$$
\sigma_{b}^{a}=\left[\sum_{i=1}^{N}\left(f_{a}\left(g_{i}\right)-f_{b}\left(g_{i}\right)\right)^{2}\right]^{1 / 2}
$$

where the sum was taken over a $5^{\circ} \times 5^{\circ} \times 5^{\circ}$ grid in a region containing the fundamental region of Euler space for cubic symmetry defined by $0^{\circ} \leq \varphi_{1} \leq 360^{\circ}$, $55^{\circ} \leq \Phi \leq 90^{\circ}$ and $0^{\circ} \leq \varphi_{2} \leq 90^{\circ}$.

\section{RESULTS}

Pole figures (see Figure 1) were constructed from the single orientation measurements. The pole figures exhibit the rolling texture as expected.

Figure 2 displays $\Phi=75^{\circ}$ section plots for ODFs calculated using both odd and even coefficients, $f_{N}(g)$, ODFs calculated using only even coefficients, $f_{N}^{(0)}(g)$, and a first order correction, $f_{N}^{(1)}(g)$, of the incomplete ODF. It should be noted that the main components of the texture appear after only 100 measurements. Only a first-order correction is shown as it was found that it gave a $9.1 \%$ correction, whereas, successive iterations gave only $1.4 \%$ and $0.3 \%$ corrections.

Figure 3 contains the $\Phi=55^{\circ}-90^{\circ}$ sections of the ODF calculated using the classical pole figure approach without correction, $f_{\text {pole } f .}(g)$, as obtained from the $\mathrm{X}$-ray diffraction measurement and the complete ODF calculated using 1000 measurements, $f_{1000}(g)$.
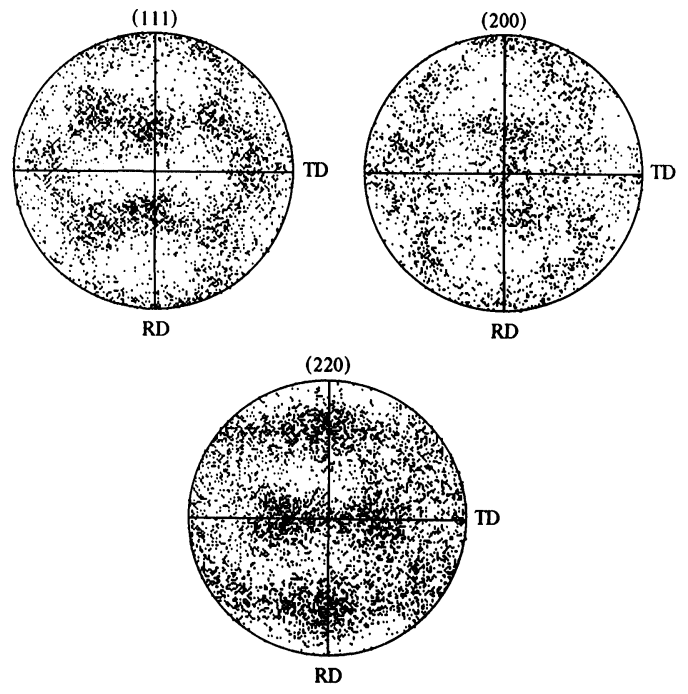

Figure 1 Pole figures obtained from single orientation measurements. 


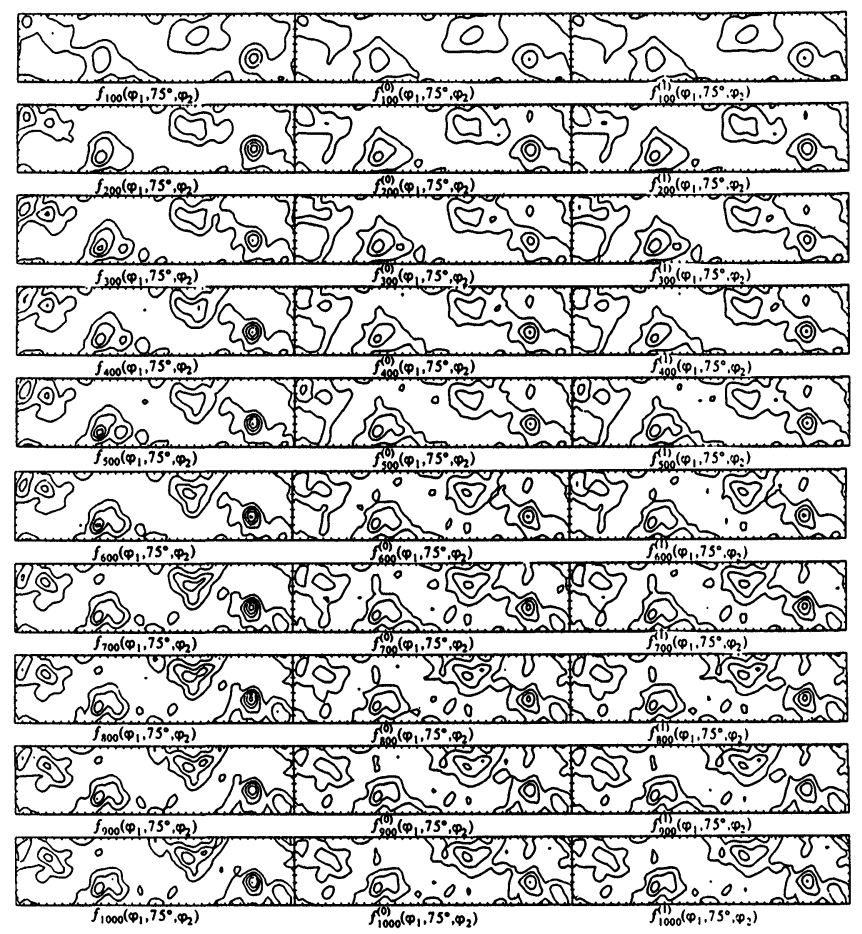

Figure $2 \Phi=75^{\circ}$ section plots of $f_{N}(g), f_{N}^{(0)}(g)$ and $f_{N}^{(1)}(g)$ for $N=100,200, \ldots, 1000$ measurements. Intensity levels at $1,3,5,7,9$. Long axis $=\varphi_{1}$, short axis $=\varphi_{2}$.

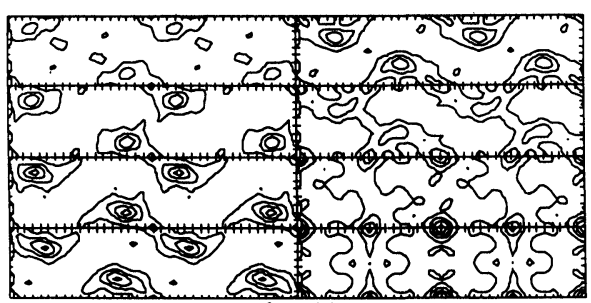

$$
f_{\text {pole } \mathrm{r}}(\mathrm{g})
$$

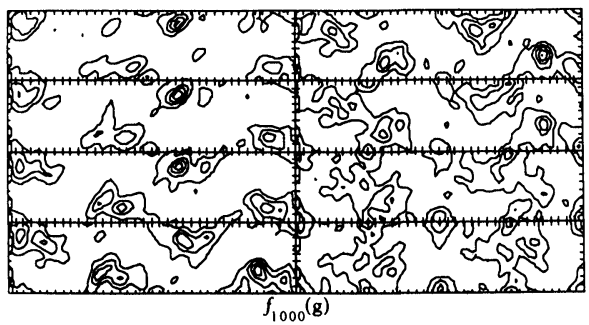

Figure $3 \Phi=55^{\circ}-90^{\circ}$ section plots of $f_{\text {polef. }}(g)$ and $f_{1000}(g)$. Intensity levels at $1,3,5,7,9$. Long axis $=\varphi_{1}$, short axis $=\varphi_{2}$. 


\section{DISCUSSION OF RESULTS}

An inspection of the plots in Figure 2 suggests that the location of the main peaks in the ODF have stabilized after only 100 measurements but the peak heights and secondary features require more measurements to stabilize. This suggests that a stronger texture would require less measurements to approximate than a more random texture. Field (1988) has shown this phenomenon in pole figures of copper tubing undergoing torsion. After $200 \%$ strain the torsion texture was evident in the pole figures after only $\mathbf{4 0}$ measurements. However, the orientations are more difficult to measure at the higher strains because the backscatter patterns become noisy due to small misorientations between sub-grains generated by the deformation. Furthermore, Van Houtte (1983) maintains that the positivity correction technique works better in strongly textured materials, so it cannot be inferred that at stronger textures the single orientation method becomes any more advantageous.

Let $f_{1000}(g)$ (the ODF calculated using both odd and even coefficients using 1000 measurements) be considered the absolute ODF for the single orientation measurements. Now let $\sigma_{N(i)}$ be the mean square error between the incomplete ODF calculated using $N$ measurements having undergone $i$ positivity correction iterations and the absolute ODF. Let $\sigma_{\text {random }}$ be the mean square error between a completely random ODF and the absolute ODF. Figure 4 shows that at approximately 750 measurements the ODF calculated using both odd and even coefficients, $f_{750}(g)$, exceeds the accuracy of the incomplete version of the absolute ODF after correction, $f_{1000}^{(1)}(g)$. This implies that an ODF calculated from 750 single orientation measurements represents a more statistically reliable approximation of the true ODF than one attainable using pole figure data; given that the ODF calculated using 1000 measurements is a statistically accurate approximation of the true ODF.

In order to ascertain the validity of using $f_{1000}(g)$ as an absolute ODF it was compared with the ODF calculated using the classical pole figure approach. The mean square errors between the ODFs calculated using 100 and 1000 measurements and the ODF calculated using the classical pole figure approach were found to be 1.89 and 0.89 . If the ODF calculated using the classical approach is assumed

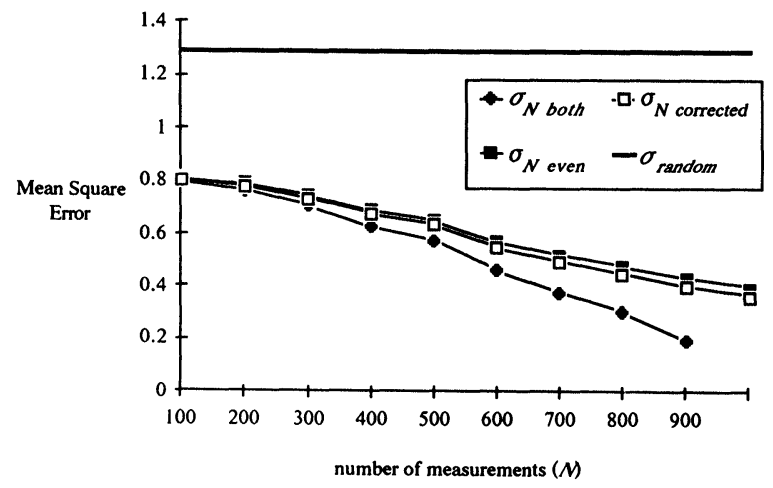

Figure $4 \sigma_{N}=\sigma_{N \text { both }}, \sigma_{N(0)}=\sigma_{N \text { even }}, \sigma_{N(1)}=\sigma_{\text {corrected }}$ for $N=100,200,300, \ldots, 1000$. 
Table 1 Locations of the recrystallization and rolling texture components

\begin{tabular}{llrrr}
\hline & Component & \multicolumn{3}{c}{ Euler angles } \\
\cline { 3 - 5 } & & $\varphi_{1}$ & \multicolumn{1}{c}{$\Phi$} & $\varphi_{2}$ \\
\hline \multirow{2}{*}{ Recrystallization } & Cube & 90 & 0 & 270 \\
Rolling & Goss & 180 & 45 & 270 \\
& Brass & 140 & 45 & 270 \\
& 2 & 135 & 40 & 280 \\
& 3 & 125 & 35 & 290 \\
& 4 & 115 & 31 & 300 \\
& 5 & 100 & 30 & 310 \\
& Copper & 90 & 30 & 315 \\
\hline
\end{tabular}

to be a better approximation of the true ODF then a simple linear interpolation of the mean square errors between $f_{100}(g)$ and $f_{1000}(g)$ and the classical ODF suggests that 1800 measurements would be needed to match the accuracy of the classical ODF.

Consider a second approach to interpreting the results. Along with the $W$ coefficients ALCOA provided the ODF values at the recrystallization and rolling texture components. The location of these components is given in Table 1.

Figure 5 shows the intensities of ODFs calculated using 100, 300, 500, 700, 900 and 1000 measurements at the texture components. It is evident that for the higher peaks it takes more measurements for the peaks to reach their true peak heights. Indeed, the plot implies that 1000 measurements does not give an accurate measurement of the actual peak heights in the ODF.

If the single orientation measurement is compared with the classical pole figure approach (see Figure 6) it is apparent that after 1000 measurements the peak associated with the strongest of the recrystallization and rolling texture components have not "filled up", suggesting that more measurements are needed to exactly fix the peak heights.

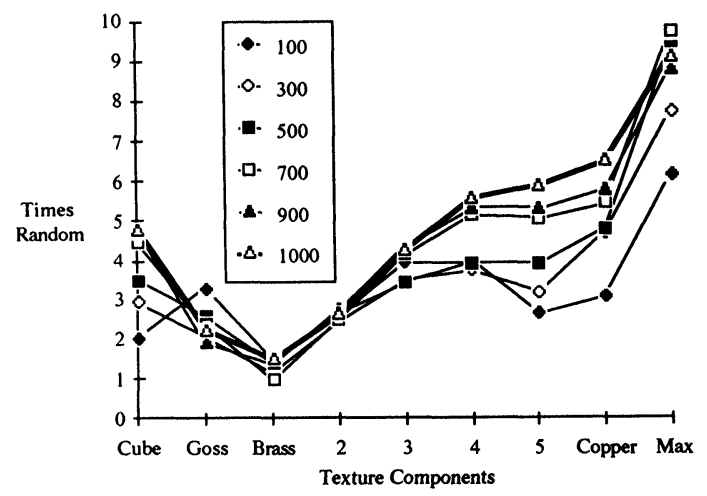

Figure 5 Intensities of the recrystallization and rolling texture components for ODFs calculated using $100,300,500,700,900$ and 1000 measurements. 


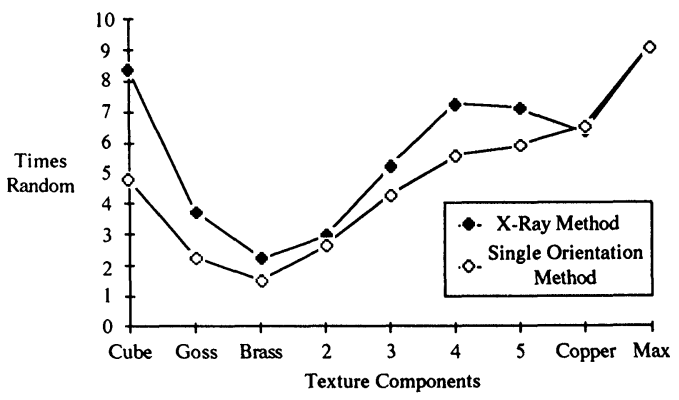

Figure 6 Intensities of the recrystallization and rolling texture components for $f_{\text {polef. }}(g)$ and $f_{1000}(g)$.

A local maxima study was made to insure that this phenomena was not due to a shift in actual peak locations due to a misalignment in the phsyical experiments. Another factor that could contribute to the difference was the half-width of the Gaussian peak, $\Phi_{0}$, used in the calculation of the $C$ coefficients per Eqs (3) and (4). $\Phi_{0}$ did contribute to the height of the peaks as shown in Figure 7; nevertheless, the effect was not strong enough to overcome the discrepancy in peak heights without introducing additional error as the Gaussian approached a delta function.

Figure 8 provided a means for predicting how many measurements would be needed to "fill up" the peaks of Figures 5 and 6 . The function $\Lambda(N)$ in the plot is given by:

$$
\Lambda(N)=(1 / 8) \sum_{i=1}^{8} \frac{f_{N}\left(g_{i}\right)}{f_{\text {polef. }}\left(g_{i}\right)}
$$

where $g_{i}$ represents the orientation of the $i$ th texture component. This plot suggests that 2400 measurements would give a statistically reliable approximation of the true ODF assuming that the height of the peaks is accurate in the ODF calculated by the traditional pole figure approach. As previously discussed less measurements are needed to approximate a true ODF for a stronger texture. This is equivalent to a steepening of the slope in Figure 8.

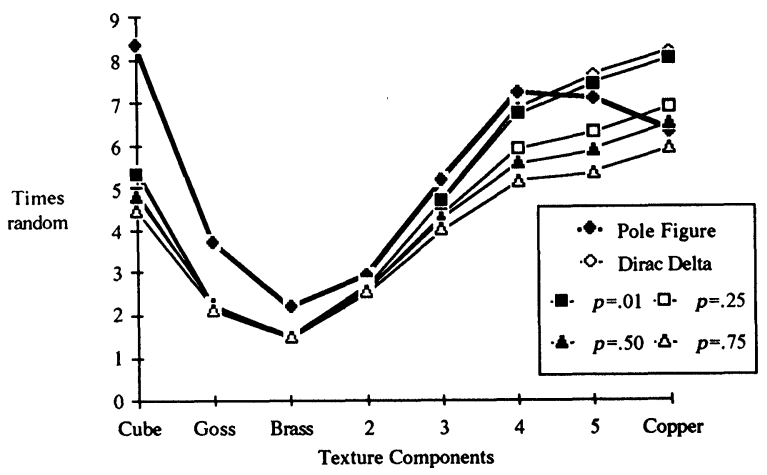

Figure 7 Intensities of the recrystallization and rolling texture components for ODFs calculated using several values of $p .(N=1000)$ and for $f_{\text {polef. }}(g)$. 


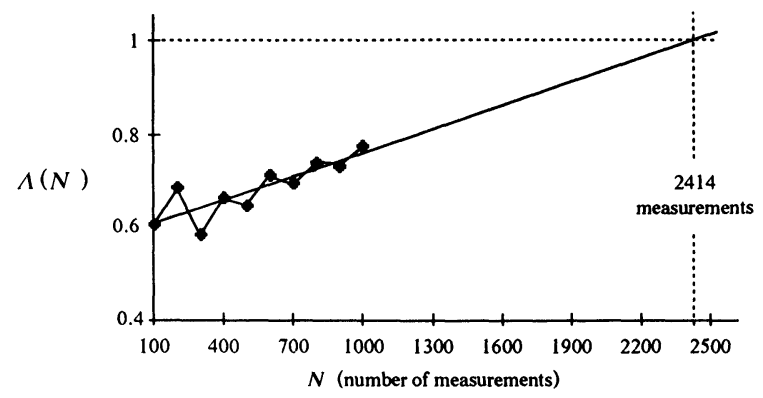

Figure 8 Plot of the average of $f_{n}(g) / f_{\text {polef. }}(g)$ over each of the texture components versus the number of measurements, $N$.

\section{SUMMARY OF STRENGTHS AND WEAKNESSES OF THE TWO METHODS}

\section{Conclusions}

The primary conclusion that can be drawn from this study is that the single orientation method provides a practical means for estimating the texture. However, if very correct quantitative data on the peak heights is required this method requires a considerable investment in experimental measurement. The time required to make enough measurements would exceed the time required to measure pole figures, especially in actual operator time. At the completion of the acquisition of the single orientation data set we were capable of measuring 150 single orientations in an hour using the Dingley system for this moderate texture. If the current measurement technique could be automated it is conceivable that this technique could be used to accurately approximate true ODFs.

\section{Pole Figure Method}

Strengths. An X-ray diffraction experiment requires a smaller investment in time than the single orientation method. Another advantage is that X-rays sample by volume reducing the chance for surface effects to dominate a texture experiment.

Weaknesses. The pole figure method furnishes only indirect access to the odd $C$ coefficients through correction schemes. Another disadvantage is that low symmetry materials require very many pole figures to facilitate an accurate harmonic expansion of the ODF.

\section{Single Orientation Method}

Strengths. The single orientation method gives direct access to both the odd and even coefficients. This not only improves the accuracy of the ODF calculation, but more importantly, the dependability of properties' models which require the odd coefficients such as piezoelectricity and pyroelectricity (Johnson and Ferrari, 1988). If a systematic measurement array is used then not only is orientation distribution information available but information concerning the orientation 
correlation of neighboring crystallites is also inherently contained in the data. Not only is orientation information available in the backscattering patterns, but the width of the bands in the patterns provides a means of measuring the local elastic strain fields as well.

Weaknesses. The primary weakness of the single orientation method is that it requires a considerable investment in operator time to collect data unless the identification of the patterns can be automated. A second problem is that there may be some systematic biasing in the sampling method; for example, areas of high lattice curvature give fuzzy pictures which cannot be positively identified and may be excluded in the single orientation method but included in the pole figure method.

\section{ACKNOWLEDGEMENT}

Funding for this research was provided by the Division of Materials Research of the National Science Foundation under grant DMR-8896259 and by the Aluminum Company of America.

\section{References}

Bunge, H. J. (1965). Z. Metallkde. 56, 872.

Bunge, H. J. (1982). Texture Analysis in Materials Science. Butterworths, London.

Dahms, M. and Bunge, H. J. (1988). ICOTOM 8, Proc. of the Eighth Int. Conf. on Textures of Materials, Santa Fe, p. 79.

Dingley, D. J. (1988). ICOTOM 8, Proc. of the Eighth Int. Conf. on Textures of Materials, Santa Fe, p. 189.

Field, D. (1988). MS Thesis, BYU-Provo, Utah.

Imhof, J. (1982). Textures and Microstructures 4, 189.

Johnson, G. C. and Ferrari, M. (1988). ICOTOM 8, Proc. of the Eighth Int. Conf. on Textures of Materials, Santa Fe, p. 115.

Lücke, K., Pospiech, J., Virnich K. H., Flemmer, A. and Nüssler, D. Published partly in: Flemmer A.; Thesis, RWTH-Aachen (1976), Nüssler D.; Diplom Thesis, RWTH-Aachen (1977), Virnich K. H.; Diplom Thesis, RWTH-Aachen (1976).

Lücke, K., Pospiech, J., Virnich, K. H. and Jura, J. (1981). Acta Met. 29, 167.

Matthies, S. and Vinel, G. W. (1982). Physica status solidi (b) 112, K111.

Matthies, S. (1979). Physica status solidi 92, K135.

Roe, R.-J. (1965). J. Appl. Phys. 36, 2024.

Ruer, D. (1976). These d'Etat, Univ. Metz.

Vadon, A. (1981). These, Univ. Metz.

Van Houtte, P. (1983). Textures and Microstructures 6, 1.

Wagner, F., Wenk, H. R., Esling, C. and Bunge, H. J. (1981). Phys. Stat. Sol(a) 67, 269.

Williams, R. O. J. Appl. Phys. 39, 4329 (1968a).

Williams, R. O. (1968b). Trans. Met. Soc. AIME 242, 104. 